\title{
Chemical Analysis of Airbrushed Nickel Nanoparticles for Large-Area Growth of Vertically Aligned Carbon Nanofibers on Metal Surfaces
}

\author{
Adedapo Oni ${ }^{1}$, Ryan White ${ }^{1}$, Mehmet Sarac ${ }^{1}$, Bryan Anderson ${ }^{1}$, Ryan Pearce ${ }^{1}$, Justin Railsback ${ }^{1}$, Dale \\ Hensley $^{2}$, Anatoli Melechko ${ }^{1}$, Joseph Tracy ${ }^{1}$, James LeBeau ${ }^{1}$ \\ 1. North Carolina State University, Materials Science and Engineering, Raleigh, USA. \\ 2. Oak Ridge National Laboratory, Oak Ridge, USA.
}

Vertically aligned carbon nanofibers (VACNFs) are of critical interest in applications including electrochemical probes [1], gene delivery devices [2], and batteries [3]. Typically, VACNFs are grown by catalytic nanoparticles (NPs) distributed on $\mathrm{Si}$ substrates where $\mathrm{SiN}_{\mathrm{x}}$ coatings on the VACNFs provide enhanced structural support. In this case, the $\mathrm{Si}$ for nitride formation is provided by the substrate. Growth on metals surface overcomes many integration challenges with the comparatively brittle Si substrates [3]. For example, VACNFs grown on $\mathrm{Cu}$ substrates hold potential for use as an anode material in Li-ion batteries. For VACNFs mechanical rigidity on metal substrates, however, Si micropowder is added to promote the formation of $\mathrm{SiN}_{\mathrm{x}}$ during VACNF growth [1]. Complete understanding of the final chemistry requires the analysis of VACNFs at the nanoscale with state-of-theart microscopy spectroscopy techniques.

In this talk, we report chemical analysis of VACNFs grown from airbrushed Ni nanoparticles (NPs) on $\mathrm{Al}, \mathrm{Cu}$, and $\mathrm{Ti}$ substrates using aberration-corrected scanning transmission electron microscopy (FEI Titan G2 60-300 S/TEM) equipped with a state-of-the-art Super-X EDS detector system. We will show that the high sensitivity Super-X detector system enables dramatic insight into VACNF growth behavior through simultaneous chemical analysis by EDS mapping for all of the constituent elements.

Figure 1 presents a schematic illustration of the method for patterning Ni NPs and growing VACNFs by plasma-enhanced chemical vapor deposition (PECVD). From these results, we will demonstrate that important information about the structure and composition of the interior and exterior of the vertically aligned carbon nanofibers (VACNFs) grown on different metal substrates can be rapidly determined. Furthermore, we will show elemental mapping of VACNFs grown on an Al substrate that reveal the formation of a $\mathrm{SiN}_{\mathrm{x}}$ coating encapsulating the VACNF, and the position of Ni NPs at the fiber tips, as shown in Figure 2. Finally, we will conclude with discussing the prospects for catalyst NPs deposited via airbrushing as an economically viable method to pattern catalytic growth over large areas. 
References:

[1] Melechko et al, Nanotechnology 14 1029-1035 (2003).

[2] McKnight et al, M. L. Nano Lett. 4 1213-1219 (2004).

[3] Tse et al., J. Chem. Mater. 19 5734-5741 (2007)

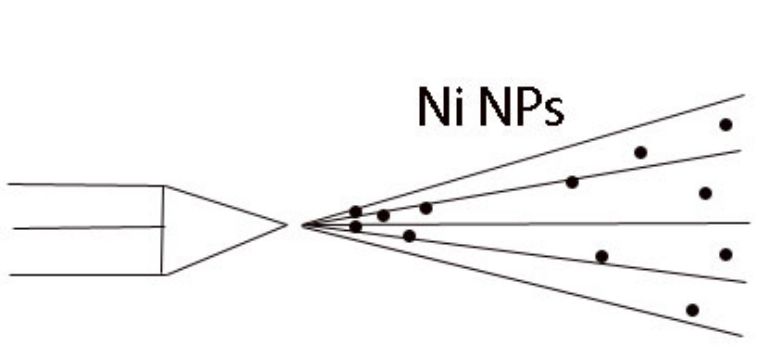

Airbrush gun
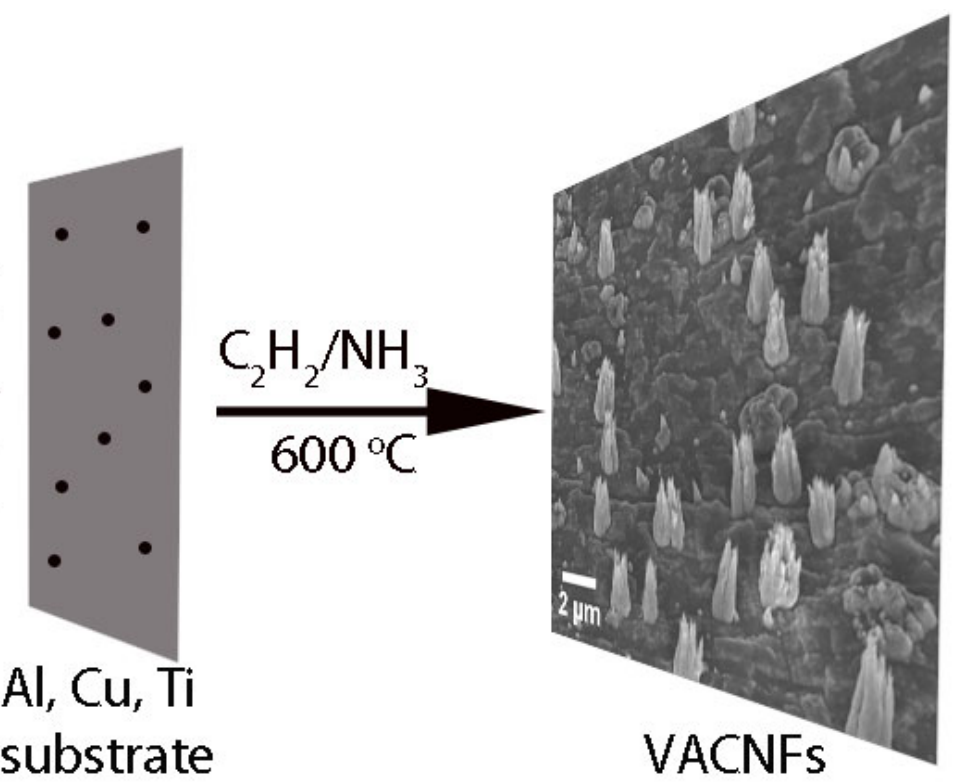

Figure 1: Schematic illustration of the method for patterning Ni NPs and growing VACNFs by plasmaenhanced chemical vapor deposition.

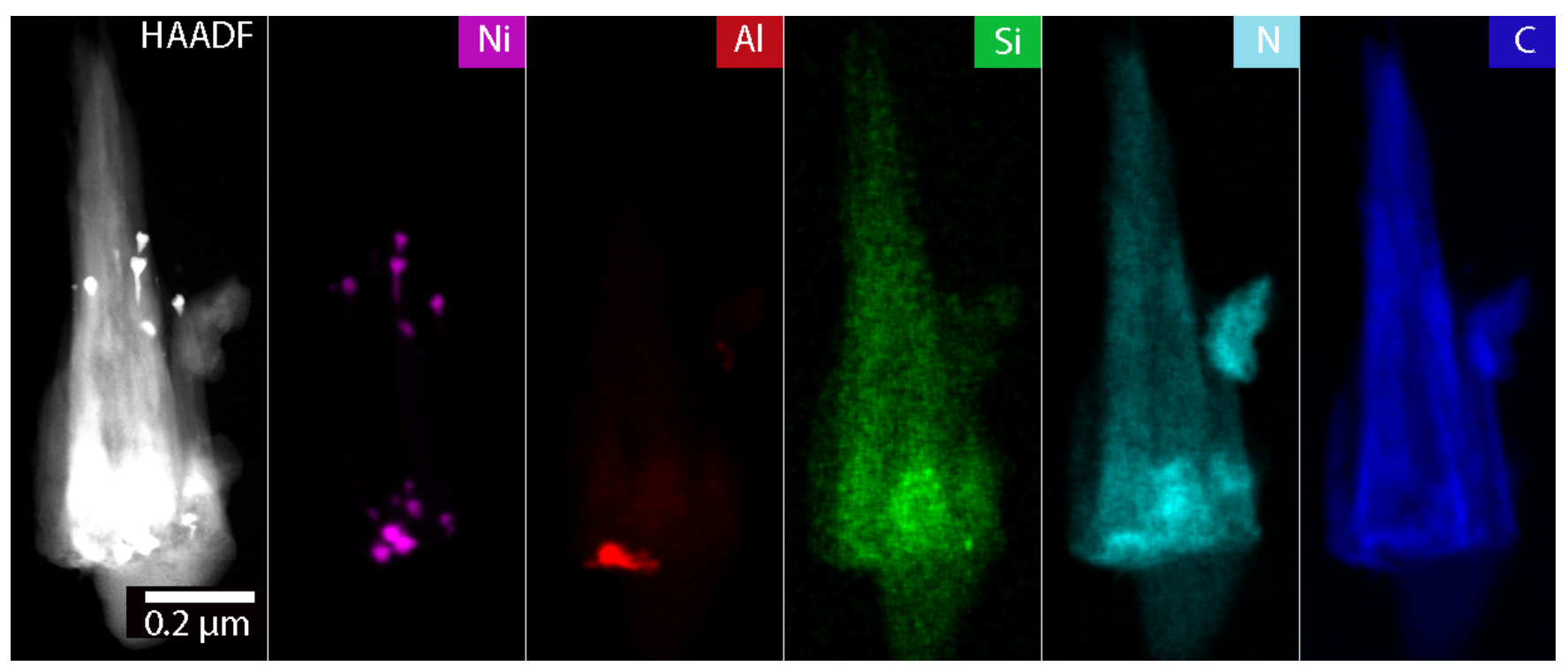

Figure 2: EDS mapping of VACNFs grown on Al foil. Elemental mapping reveals formation of a $\operatorname{SiN}_{x}$ coating encapsulating the VACNFs, which provides structural support to the nanofiber. 